ARTICLE

DOI: $10.1038 / \mathrm{s} 41467-017-02397-0$

\title{
Allosteric modulation of protein-protein interactions by individual lipid binding events
}

Xiao Cong ${ }^{1,3}$, Yang Liu¹, Wen Liu (1) ${ }^{1}$, Xiaowen Liang ${ }^{1} \&$ Arthur Laganowsky ${ }^{1,2}$

The diverse lipid environment of the biological membrane can modulate the structure and function of membrane proteins. However, little is known about the role that lipids play in modulating protein-protein interactions. Here we employed native mass spectrometry (MS) to determine how individual lipid-binding events to the ammonia channel (AmtB) modulate its interaction with the regulatory protein, GlnK. The thermodynamic signature of AmtB-GInK in the absence of lipids indicates conformational dynamics. A small number of lipids bound to $A m t B$ is sufficient to modulate the interaction with GlnK, and lipids with different headgroups display a range of allosteric modulation. We also find that lipid chain length and stereochemistry can affect the degree of allosteric modulation, indicating an unforeseen selectivity of membrane proteins toward the chemistry of lipid tails. These results demonstrate that individual lipid-binding events can allosterically modulate the interactions of integral membrane and soluble proteins.

\footnotetext{
${ }^{1}$ Institute of Biosciences and Technology, Texas A\&M Health Science Center, Houston, TX 77030, USA. ${ }^{2}$ Department of Chemistry, Texas A\&M University, College Station, TX 77843, USA. ${ }^{3}$ Present address: Wolfe Laboratories Inc., 19 Presidential Way, Woburn, MA 01801, USA. Correspondence and requests for materials should be addressed to A.L. (email: alaganowsky@chem.tamu.edu)
} 
T he regulated transport of ions and solutes across biological membranes is an essential chemical process. Regulation of ion channels is carried out by their molecular interactions with regulatory proteins, small molecules, lipids, or through posttranslational modification ${ }^{1-4}$. Although some channels have been shown to be regulated by specific lipid-protein interactions, such as potassium channels by phosphoinositides ${ }^{5}$, beyond these specific examples we have very limited knowledge of how the chemically diverse lipid environment of the biological membrane modulates the structure and function of membrane protein.

As a step toward studying membrane protein and soluble protein interactions, we selected for study the ammonium channel (AmtB) from Escherichia coli (E. coli), an integral membrane protein that is regulated by the soluble trimeric protein $\mathrm{GlnK}^{6,7}$. The interaction between $\mathrm{AmtB}$ and $\mathrm{GlnK}$ is tightly controlled by cellular nitrogen status through key effector molecules, while adenosine diphosphate (ADP) alone is sufficient to form the complex ${ }^{8-10}$. Atomic structures have revealed that GlnK blocks ammonia transport by essentially plugging the conducting channel within each subunit primarily through the insertion of a long surface loop (Supplementary Fig. 1) ${ }^{11}$, 12. Importantly, no lipids are resolved in the crystal structures of the AmtB-GlnK complex. Along these lines, there are 19 additional atomic structures of AmtB deposited in the Protein Data Bank ${ }^{13}$. For one of the crystal structures of $A m t B$, native mass spectrometry (MS) was used to identify a lipid that stabilized the channel, which guided co-crystallization trails leading to the first structure of $\mathrm{AmtB}$ bound to lipid ${ }^{14}$. However, for the other structures no bound lipids were observed, implying that crystallography will not always be adequate in the identification and biophysical characterization of lipids involved in membrane-protein interactions, emphasizing the necessity for new techniques to investigate protein-lipid interactions.
To determine how individual lipid-binding events modulate the interaction of AmtB with GlnK, we employed native MS, a powerful biophysical technique that has emerged over the past two decades to study proteins and their interactions with ligands ${ }^{15-20}$. Unlike other biophysical techniques, which are often ensemble measurements, native MS can preserve non-covalent interactions in the gas-phase as well as resolve and interrogate individual ligand-binding events to protein complexes ${ }^{19-22}$. Advances in native MS spanning nearly a decade ${ }^{23}$ have led to the ability to study membrane protein complexes in a native-like state that can provide invaluable information on their interactions with ligands, such as nucleotides, drugs, peptides, and lipids, and subunit stoichiometry ${ }^{14,24-29}$. Recently, native MS coupled with a temperature-controlled source has been applied to monitor thermal unfolding ${ }^{30}$ as well as determine thermodynamics for protein-ligand interactions, including membrane protein-lipid interactions $^{31}$. Importantly, thermodynamic parameters for soluble protein-ligand interactions determined using native MS are in agreement with those obtained using other biophysical techniques, such as isothermal titration calorimetry and surface plasmon resonance $(\mathrm{SPR})^{31,32}$. Building upon these recent advances, we use native MS to reveal that individual lipid-binding events allosterically modulate integral membrane protein and soluble protein interactions.

\section{Results}

Biophysical characterization of the AmtB-GlnK complex. We recorded a mass spectrum for the AmtB-GlnK complex in ammonium acetate buffer containing $50 \mu \mathrm{M}$ ADP and two times the critical micelle concentration of tetraethylene glycol detergent $\left(\mathrm{C}_{8} \mathrm{E}_{4}\right)$, a detergent that exhibits charge reducing properties ${ }^{33}$, and at a set temperature of $298 \mathrm{~K}$ using a temperature-controlled
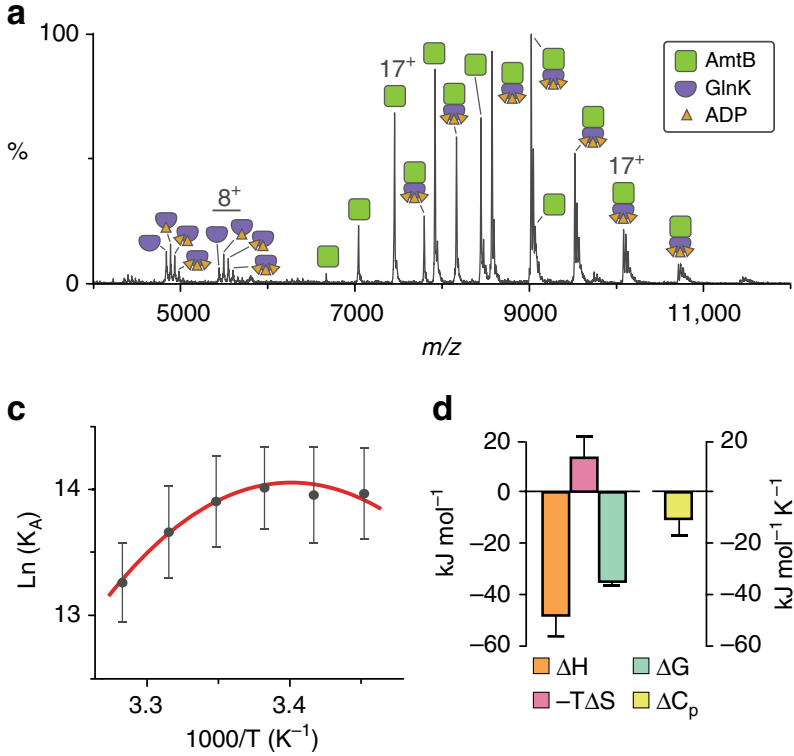
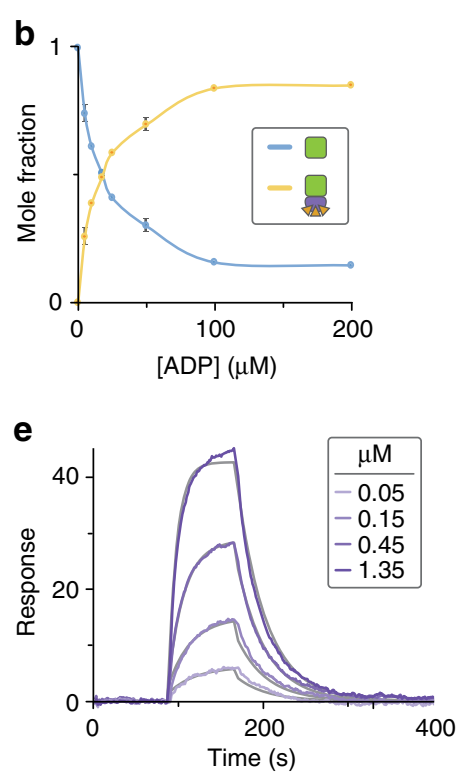

Fig. 1 Biophysical characterization of the AmtB-GInK complex by native MS and surface plasmon resonance. a Native mass spectrum of AmtB-GInK at $2 \mu \mathrm{M}$ in the presence of $50 \mu \mathrm{M}$ ADP. $\mathbf{b}$ The effect of ADP concentration on GInK association with AmtB determined by native MS. Plot of the mole fraction of AmtB and AmtB-GInK as a function of total ADP concentration. Reported are the average and s.e.m. from repeated measurements $(n=2)$. c van't Hoff plot for AmtB-GInK binding in buffer containing $50 \mu \mathrm{M}$ ADP (gray dots) as determined by native mass spectrometry and resulting fit $\left(R^{2}=0.98\right.$ ) of the nonlinear "van't" Hoff equation (red line). Reported are the average and s.e.m. from repeated measurements $(n=4)$. $\mathbf{d}$ Thermodynamic signature of AmtB-GInK binding derived from native MS in the absence of lipid at $298 \mathrm{~K}$. Reported are the average and s.e.m. from repeated measurements $(n=4)$. The change in free energy $(\Delta G)$ was calculated directly from repeated measurements of $K_{D, A G}$ at $298 \mathrm{~K}$, and entropy $(\Delta S)$ was back calculated using both $\Delta H$ and $\Delta G$ (Supplementary Table 1). e Representative sensorgrams of AmtB injected at different concentrations over a sensor surface immobilized with GlnK at $298 \mathrm{~K}$ (purple lines) and resulting fit of a Langmuir 1:1 binding model (gray lines). Reported are the average and s.e.m. from repeated measurements ( $n=4$ ) 
source $^{31}$. The mass spectrum revealed an equilibrium of GlnK, $A m t B$, and AmtB-GlnK (Fig. 1a). Around $5000 \mathrm{~m} / z$, we observed signals corresponding to apo and $\mathrm{ADP}_{1-3}$ bound states of $\mathrm{GlnK}$. The mole fraction of $\mathrm{GlnK}(\mathrm{ADP})_{0-3}$ in the mass spectrum is in agreement with those calculated using reported equilibrium dissociation constants $\left(K_{\mathrm{D}}\right)$ for GlnK-binding $\mathrm{ADP}_{1-3}$ in the absence of detergent (Supplementary Fig. 2) ${ }^{31}$. In addition, the measured mass for AmtB-GlnK is in agreement with three molecules of ADP bound. However, under our experimental conditions, we did observe a small signal corresponding to an additional ADP bound to both $A m t B-G \ln K$ and AmtB. This is likely non-specific adduction due to the high concentration of ADP and dissipates with lower ADP concentrations. These non-specific adducted peaks were taken into account when calculating the mole fraction of $\mathrm{AmtB}$ and $\mathrm{AmtB}-\mathrm{G} \operatorname{lnK}$, and we determined the equilibrium dissociation constant for GlnK-binding $\mathrm{AmtB}\left(K_{\mathrm{D}, \mathrm{AG}}\right)$ to be 1.12 $\pm 0.41 \mu \mathrm{M}$ in the presence of $50 \mu \mathrm{M}$ ADP.

To investigate further the influence of ADP concentration on GlnK binding to AmtB, we recorded mass spectra for a titration series of ADP and determined $K_{\mathrm{D}, \mathrm{AG}}$ in a similar fashion described in the preceding paragraph (Fig. $1 \mathrm{~b}$ and Supplementary Fig. 3). The $K_{\mathrm{D}, \mathrm{AG}}$ varied from $3.8 \mu \mathrm{M}$ at the lowest $\mathrm{ADP}$ concentration $(5 \mu \mathrm{M})$, whereas at the highest concentration of $\operatorname{ADP}(200 \mu \mathrm{M})$ the equilibrium-binding constant dropped to $77.68 \mathrm{nM}$. At and above an ADP concentration of $100 \mu \mathrm{M}$, the $K_{\mathrm{D}, \mathrm{AG}}$ approaches an asymptote around $\sim 80 \mathrm{nM}$ (Supplementary Fig. 3). From these experiments, a concentration of $50 \mu \mathrm{M}$ ADP was selected to give an equilibrium of $A m t B$ and $A m t B-G \ln K$ in approximate equal abundance, which is ideal for probing the effects of temperature and lipid binding (discussed below) on the AmtB-GlnK equilibrium-binding constant and was thus used in the following studies.

To determine the molecular forces behind the molecular recognition of $\mathrm{GlnK}$ by $\mathrm{AmtB}$, we recorded mass spectra at a fixed ADP concentration $(50 \mu \mathrm{M})$ and different temperatures. We then performed a "'van't"' Hoff analysis ${ }^{34}$ to extract the thermodynamics for the AmtB and $G \ln K$ interaction. A plot of the natural $\log$ of $K_{\mathrm{D}, \mathrm{AG}}$ as a function of the reciprocal of temperature was initially analyzed by fitting the "van't" Hoff equation ${ }^{34}$ to the data, which resulted in a poor fit $\left(R^{2}=0.68\right)$. In contrast, using the nonlinear form of "van't" Hoff equation ${ }^{35}$ yielded exceptional fits with an $R^{2}$ of 0.98 , indicating that heat capacity is not constant over the selected temperature range (for review see ref. ${ }^{36}$ ). Using the nonlinear van't Hoff equation enabled us to determine the change in heat capacity $\left(\Delta C_{\mathrm{p}}\right)$ and change in enthalpy $(\Delta H)$ at a reference temperature of $298 \mathrm{~K}$ (Fig. 1c, d). The change in free energy $(\Delta G)$ was calculated directly from repeated measurements of $K_{\mathrm{D}, \mathrm{AG}}$ at $298 \mathrm{~K}$, and entropy $(\Delta S)$ was back calculated using both $\Delta H$ and $\Delta G$ (Supplementary Table 1). In short, the nonlinearity in the "van't" Hoff plot suggests significant temperature-dependent conformational changes ${ }^{36}$ in AmtB and/ or GlnK.

To corroborate our findings by native MS, we used SPR with GlnK immobilized on the sensor surface. Sensorgrams for different concentrations of $\mathrm{AmtB}$ were recorded at different temperatures in the same buffer and detergent used for native MS studies (Fig. 1e and Supplementary Fig. 4). Fitting the data to a Langmuir 1:1 binding model resulted in a $k_{\text {on }}$ of $5.49 \times 10^{4} \mathrm{M}^{-1}$ $\mathrm{s}^{-1}$ and $k_{\text {off }}$ of $3.14 \times 10^{-2} \mathrm{~s}^{-1}$ (Supplementary Table 1). The average $K_{\mathrm{D}, \mathrm{AG}}$ was determined to be $0.65 \mu \mathrm{M}$, which is in accord with that measured by native MS. We also observed similar nonlinear "van't" Hoff plots for measurements obtained using SPR (Supplementary Fig. 4). Performing a similar analysis used for native MS data, we obtained similar thermodynamic parameters from SPR measurements substantiating our native MS findings (Supplementary Table 1).
Thermodynamic parameters elucidated by native MS and SPR provide additional insight into the molecular driving forces that drive association of $\mathrm{AmtB}$ and GlnK. The AmtB-GlnK interaction is largely driven by enthalpy, which is consistent with the crystal structures of the complex ${ }^{11,12}$ where a total of 39 hydrogen bonds, six of which are mediated through water bridges, are formed between the trimeric assemblies (Supplementary Fig. 1). In addition, there is a large interface area of $2704.8 \AA^{2}$ formed between AmtB and GlnK (PDB 2NS1) ${ }^{37}$. The large negative $\Delta C_{\mathrm{p}}$ yields an upward curvature to $\Delta G$ with lower temperature ranges having smaller $K_{\mathrm{D}, \mathrm{AG}}$ values. In addition, this $\Delta C_{\mathrm{p}}$ value also suggests that these macromolecules adopt a flexible conformation or a variety of interconverting conformations ${ }^{38}$. Different conformations are supported by crystallographic structures, where a conformational change in $\mathrm{AmtB}$ is observed when bound to $\mathrm{GlnK}$ that arises from rigid body movements of several transmembrane helices within each subunit (Supplementary Fig. 1c) ${ }^{11,12}$. Moreover, negative $\Delta C_{\mathrm{p}}$ values are observed for specific protein-DNA interactions ${ }^{39}$, indicating the $\mathrm{AmtB}-\mathrm{GlnK}$ interaction is highly specific. The sign of $\Delta C_{\mathrm{p}}$ also suggests a change in polar solvation ${ }^{36,40}$, which is consistent with desolvation of the exposed T-loop of GlnK upon insertion into the ammonia conducting channels of AmtB subunits. Although deciphering the contribution of conformation equilibria and changes in solvation in $\Delta C_{\mathrm{p}}$ warrants further study, the thermodynamic parameters indicate the molecular interaction between $A m t B$ and $G \operatorname{lnK}$ is driven by enthalpy and that they adopt flexible conformations.

Lipids with different headgroups binding to AmtB-GlnK. To address whether individual lipid-binding events to AmtB could indirectly influence the interaction with $\mathrm{G} \ln \mathrm{K}$, we used native MS since it has the ability to resolve individual lipid-binding events unlike other biophysical techniques. Similar to the studies above, the mass spectrometer was tuned to obtain resolved mass spectra while minimizing activation of the complex thereby preserving non-covalent interactions (Supplementary Fig. 5). Mass spectra were recorded under optimized instrument settings for the AmtB-GlnK complex titrated with cardiolipin (TOCDL, 1,1',2,2' tetraoleoyl-cardiolipin), phosphatidic acid (PA), phosphatidylethanolamine (PE), phosphatidylglycerol (PG), phosphatidylserine (PS), or phosphatidylcholine (PC) containing 1palmitoyl-2-oleoyl (PO, 16:0-18:1) tails (Fig. 2 and Supplementary Fig. 6). Although POPC is not native to E. coli membranes, it was included since it is a commonly used lipid. Again, an ADP concentration of $50 \mu \mathrm{M}$ was selected to yield an approximate equal abundance of species enabling simultaneous measurements of lipid-binding events to both AmtB and AmtB-GlnK. The addition of lipids to the $A m t B-G \operatorname{lnK}$ complex resulted in lipids binding to both AmtB and AmtB-GlnK (Fig. 2a and Supplementary Fig. 6). Importantly, no lipid-binding events to GlnK were observed in all lipid titrations indicating that the observed lipid-binding events to $A m t B$ and $A m t B-G \operatorname{lnK}$ are specific. Equilibrium-binding constants were determined by deconvoluting mass spectra ${ }^{41}$ recorded for AmtB-GlnK titrated with different lipids and fitting the data globally to an equilibriumcoupled AmtB-GlnK lipid-binding model (Fig. 3, Supplementary Fig. 7, and Supplementary Table 2). Importantly, for all lipids, the $K_{\mathrm{D}, \mathrm{AG}}$ values were similar to our previous measurement in the absence of lipid, validating our equilibrium AmtB-GlnK lipid model (Fig. 3).

A range of modulation of the equilibrium dissociation constant $\left(K_{\mathrm{D}, \mathrm{AL} n \mathrm{G}}\right)$ for $\mathrm{AmtB}$ bound to $n$ lipids $(\mathrm{AL} n)$ binding to $\mathrm{GlnK}(\mathrm{G})$ was observed (Fig. 2). POPC, POPE, and POPG exhibited a decrease in $K_{\mathrm{D}, \mathrm{AL} n \mathrm{G}}$ with each consecutive binding event. Of the 

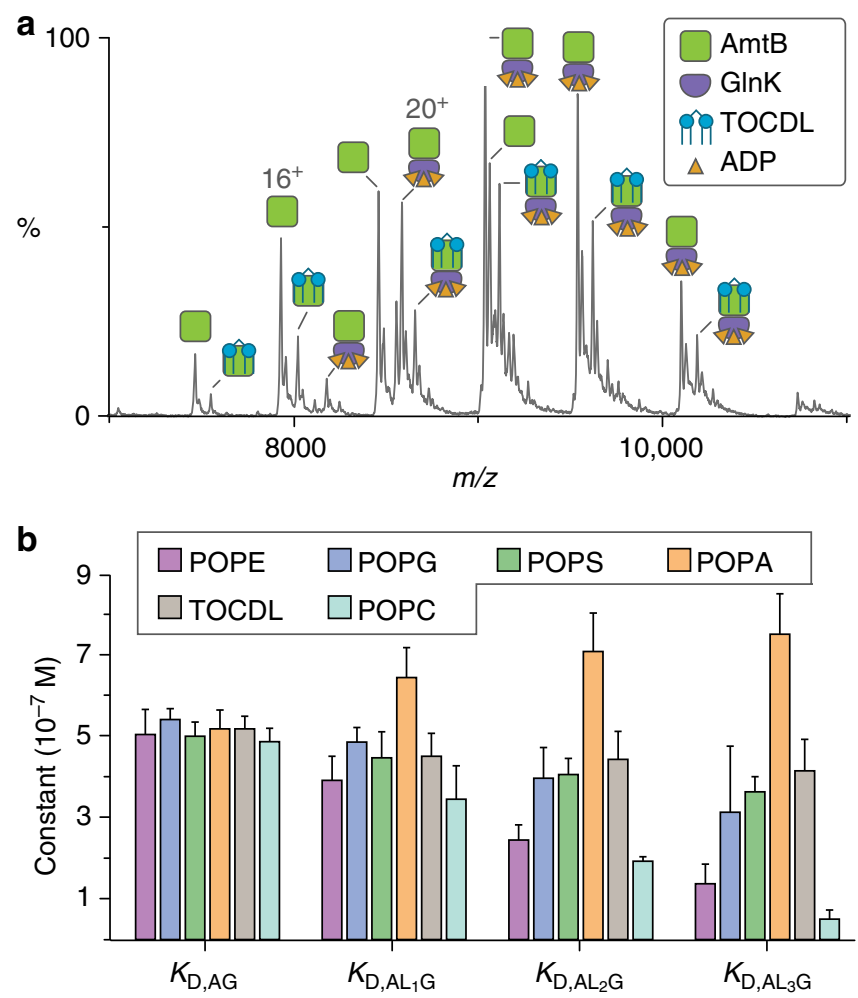

Fig. 2 Native mass spectrometry reveals individual lipid-binding events can allosteric modulate the interaction between GlnK and AmtB. a

Representative mass spectrum of AmtB-GInK at $2 \mu \mathrm{M}$ in buffer containing $50 \mu \mathrm{M}$ ADP and $20 \mu \mathrm{M}$ 1,1',2,2'-tetraoleoyl-cardiolipin (TOCDL). b Plot of equilibrium dissociation constants $\left(K_{D}\right)$ for $G \operatorname{lnK}$ binding to either apo $A m t B$ or AmtB bound to phosphatidylethanolamine (PE), phosphatidylglycerol $(P G)$, phosphatidylserine (PS), and phosphatidic acid (PA) containing 1-palmitoyl-2-oleoyl (PO, 16:0-18:1) tails, and TOCDL at 298 K (see Supplementary Fig. 7 for $K_{\mathrm{D}}$ abbreviations). Reported are the average and s.e.m. from repeated measurements $(n=3)$

three, POPC had displayed the largest decrease in $K_{\mathrm{D}, \mathrm{AL} n \mathrm{G}}$ followed by POPE. POPC differs from POPE by three methyl groups and these additional moieties likely support binding of this lipid to either a unique site or binding mode, which enhances binding to GlnK. In contrast, an opposite trend was observed for POPA with each binding event weakening the molecular interaction between $\mathrm{AmtB}$ and GlnK. On the other hand, TOCDL and POPS had little effect on $K_{\mathrm{D}, \mathrm{AL} n \mathrm{G}}$. Taken together, these results demonstrate that individual lipid-binding events can indirectly affect the molecular interaction between $\mathrm{AmtB}$ and GlnK, presumably by their particular binding mode or position.

Lipids with different tails binding to AmtB-GlnK. As individual lipid-binding events allosterically modulate the interaction between AmtB and GlnK, we calculated the coupling factors for the different lipids investigated (see "Methods" section). For POPS and TOCDL, the coupling factors exhibited neutral allosteric modulation for all lipid-binding events recorded (Supplementary Table 3). In contrast, POPG and POPE had a consistent increase in the coupling factor with each additional binding event, displaying positive allosteric modulation. More specifically, from a linear fit to the coupling factors as a function of bound lipid yields an increase of 0.4 and 0.3 in the coupling factor per lipidbinding event for POPE and POPG, respectively (Supplementary Fig. 8). Unlike the other lipids investigated, POPA exhibited slight negative allosteric modulation with each lipid-binding event.

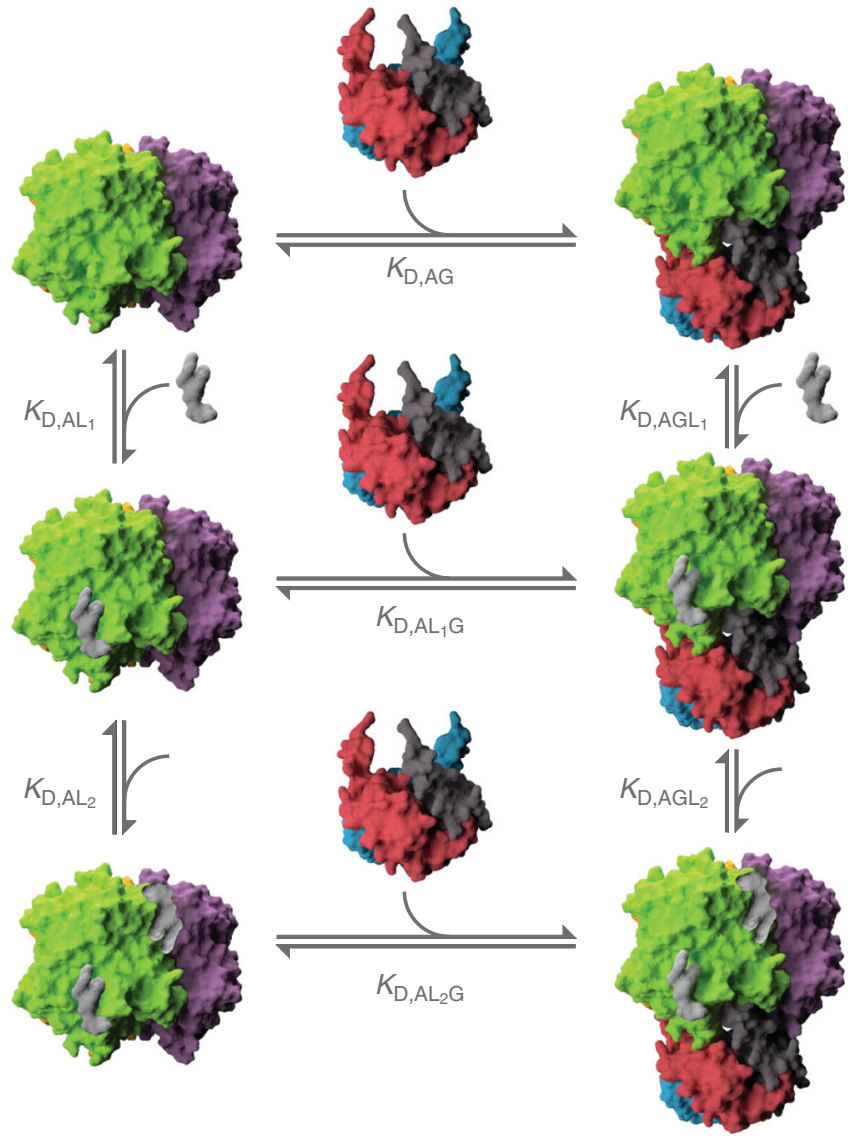

Fig. 3 Equilibrium-coupled-binding model for AmtB-binding GInK and/or lipids. AmtB (green and purple), GlnK (red, blue, and gray), and representative phospholipid (silver) are included in the model. AmtB and GInK are shown in surface representation (PDB 2NS1). ADP is not shown for clarity

Remarkably, the lipids investigated, with the exception of TOCDL, differ only by a few atoms in their headgroups yet display a range of allosteric modulation that can be detected by native MS.

Given the results for lipids with similar tails but different headgroups, we next investigated the effect of varying tail lengths of PG to allosterically modulate the AmtB-GlnK complex. PG was selected as we have previously determined the crystal structure of AmtB in complex with PG and it displayed positive allosteric modulation $^{14}$. In addition, a mutant form of $\mathrm{AmtB}$, where two residues are mutated $(\mathrm{N} 72 \mathrm{~A} / \mathrm{N} 79 \mathrm{~A})$ to abolish a specific PG site observed in the $\mathrm{X}$-ray structure, resulted in reduced gas-phase stabilization of the channel by this $\operatorname{lipid}^{14}$ and a different thermodynamic signature observed for binding $\mathrm{PG}^{31}$. Equilibrium-binding constants and coupling factors for PG lipids with different tail lengths were determined using the same procedure used for lipids with different headgroups (Fig. 4a and Supplementary Tables 4-5). Consistent with POPG, PG harboring tail lengths of 12 and 16 carbons exhibited positive allosteric modulation. Surprisingly, PG containing 14 carbon tails, in between the lipid tail lengths studied, displayed neutral allosteric modulation. This unexpected result suggests that specific PGbinding site(s) exhibit specific requirements for the lipid tails to elicit an allosteric effect.

Intrigued by the effect of lipid tail length on PG, we then studied the impact of the stereochemistry of monounsaturated carbon bonds within the lipid tails of PE on the AmtB-GInK complex. In bacteria, the stereochemistry of the monounsaturated 

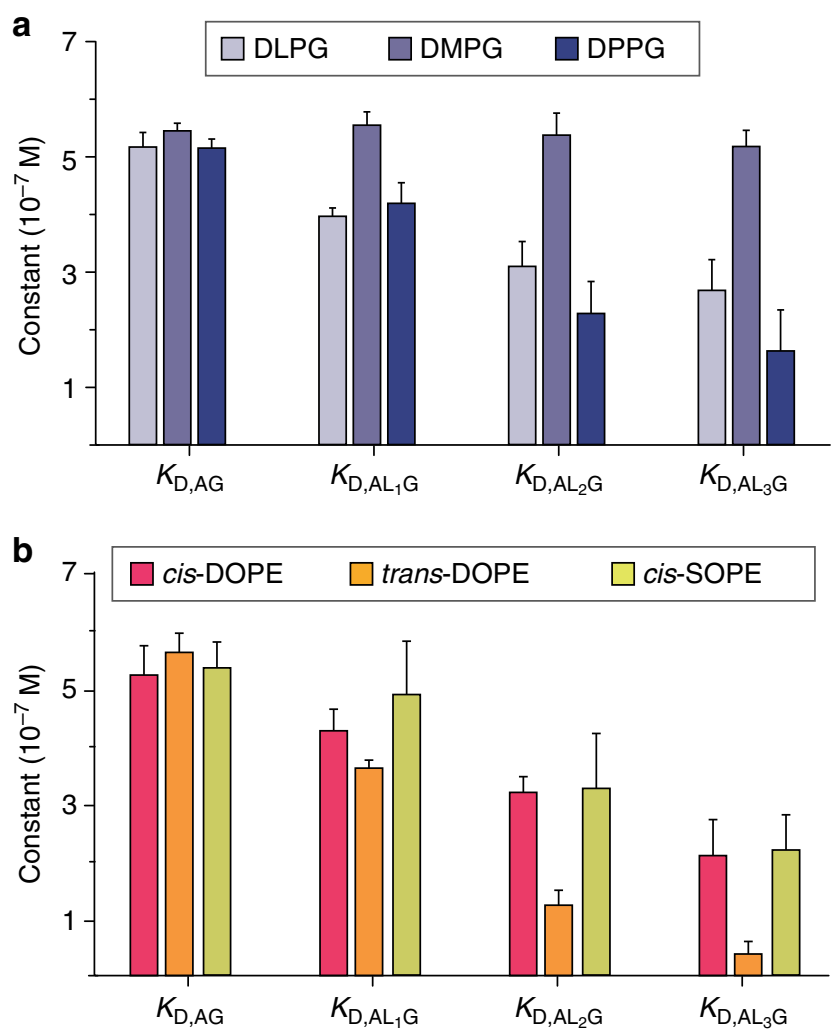

Fig. 4 Native mass spectrometry reveals the effect of lipid tail chemistry on allosteric modulation of AmtB-GlnK. a Plot of equilibrium dissociation constants $\left(K_{\mathrm{D}}\right)$ for GlnK binding apo AmtB and AmtB bound to PG lipids with increasing acyl chain length: 12 (DL, 1,2-dilauroyl), 14 (DM, 1,2dimyristoyl), and 16 (DP, 1,2-dipalmitoyl). b Plot of $K_{D}$ for GlnK binding apo $A m t B$ and $A m t B$ bound to $P E$ lipids with different stereochemistry: dioleoyl (DO, 18:1) in cis (cis-DOPE) or trans configuration (trans-DOPE), and 1stearoyl-2-oleoyl (SO, 18:0-18:1) in cis configuration (cis-SOPE). Reported are the average and s.e.m. from repeated measurements $(n=3)$

double bond is predominantly in the cis configuration, which is more abundant at lower growth temperatures, where it increases membrane fluidity 42,43 . In contrast, the trans configuration increases membrane rigidity and decreased permeability to solutes, and its abundance is increased at elevated temperatures ${ }^{43}$, ${ }^{44}$. Given the physiological relevance of lipid tail stereochemistry, the first PE lipids we investigated had dioleoyl (DO, 18:1) tails in either the cis or trans configuration. Equilibrium-binding constants and coupling factors for these lipids were determined in a similar fashion to the other lipids investigated (Fig. $4 \mathrm{~b}$ and Supplementary Tables 4-5). Interestingly, DOPE in the trans configuration, which retains a slight kinked conformation relative to saturated lipids ${ }^{43}$, displayed greater positive allosteric modulation with a coupling factor of $5.28 \pm 1.82$ for the third bound lipid, representing the largest coupling factor reported in our study (Supplementary Table 5). In contrast, the allosteric modulation for the cis configuration, which introduces a kink in the lipid tail, was not as pronounced with a coupling factor of $1.71 \pm 0.31$ for the third bound lipid. Considering that the trans configuration closely resembles that of a saturated carbon-carbon bond, we next examined PE with 1-stearoyl-2-oleoyl (SO, 18:0, 18:1), which contains saturated and cis-monounsaturated lipid tails (SOPE). The coupling factor for SOPE did not fall between the two DOPE lipids studied but was similar to cis-DOPE. Taken together, these results reveal an exquisite selectivity toward not only the headgroup of lipids, but also the length and stereochemistry of lipid tails.

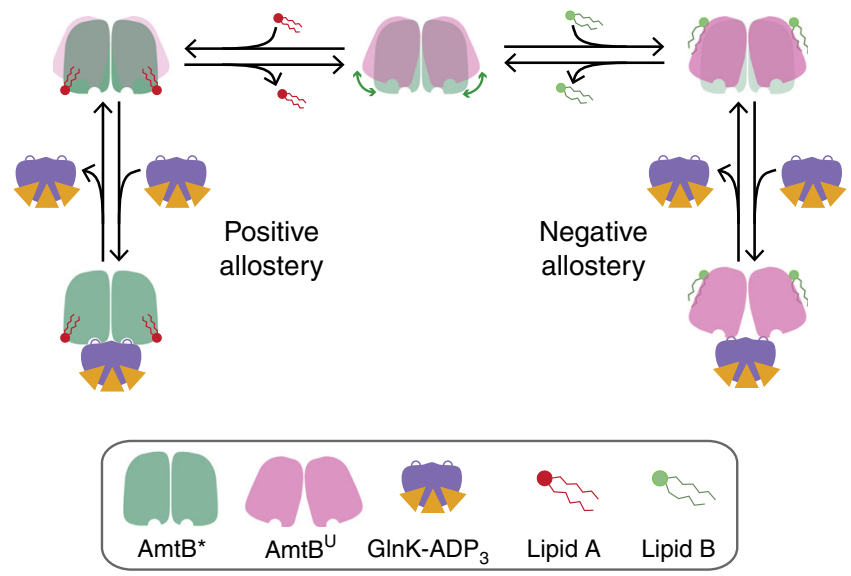

Fig. 5 A plausible model for the allosteric modulation of the AmtB-GInk by individual lipid-binding events. AmtB interconverts between two conformations (middle). Binding of specific lipids may stabilize either conformation. Those that stabilize $\mathrm{AmtB}^{\star}$, the state that resembles when bound to GInK, will lead to positive allosteric modulation. In contrast, lipids that preferentially stabilize $A m B^{U}$, the state that represents the uncomplexed conformation, will lead to reduced binding affinity to GInK or negative allostery

\section{Discussion}

Originating in $1965^{45}$, allostery has emerged as an underlying theme in biological macromolecules (for review see refs. ${ }^{46,}{ }^{47}$ ). Here we demonstrate for the first time that individual lipidbinding events can indeed allosterically modulate integral membrane and soluble protein interactions. Given that we have identified allostery, we propose a plausible mechanism to rationalize the allosteric effects observed (Fig. 5). In the simplest scenario, $\mathrm{AmtB}$ is interconverting between two conformations that are observed by X-ray crystallography (Supplementary Fig. 1c): in the uncomplexed state $\left(A m t B^{u}\right)$ or when complexed with $G \operatorname{lnK}\left(\mathrm{AmtB}^{\star}\right)$. In the case of positive allosteric modulation, binding of lipid(s) to AmtB shifts the Boltzmann distribution of the interconverting states of AmtB by preferentially stabilizing (lowering $\Delta G$ ) the $\mathrm{AmtB}^{*}$ state. As this state resembles that when bound to GlnK, it will exhibit much higher affinity for binding GlnK giving rise to positive allosteric modulation. In contrast, the negative allosteric modulation observed for POPA could be rationalized by preferentially stabilizing the $A m \mathrm{~B}^{\mathrm{u}}$ state, and since this state resembles that of the uncomplexed state, it will exhibit reduced affinity for GlnK giving rise to negative allostery.

In summary, we have shown that the interaction between $\mathrm{AmtB}$ and GlnK is associated with changes in equilibria of conformers and solvation. In addition, we demonstrate that individual lipid-binding events can influence integral membrane protein and soluble protein interactions, using the $\mathrm{AmtB}-\mathrm{GlnK}$ complex as a model system. Interestingly, lipids with similar tails but different headgroups allosterically modulate the AmtB-GlnK complex in positive, neutral, and negative fashions. The surprising result that variable lipid tail lengths of a PG and the stereochemistry of PE tails can alter the degree of allosteric modulation illustrates that specific lipid-binding site(s) are exquisitely selective for both lipid headgroup and tail. Although a small number of lipid tail lengths and stereochemistry were examined here, by extrapolation it is likely that other modifications of lipid tails will display different degrees of allosteric modulation for protein-protein interactions. Moreover, an underlying question remains regarding the location of the bound lipids and how they induce their allosteric effect. Addressing this question is a 
monumental challenge as echoed by our limited knowledge in this area. However, in our native MS studies, the lipids may be binding to specific site(s) selective for headgroup and/or lipid tails, or binding to similar non-specific site(s). In closing, this is the first report demonstrating that individual lipid-binding events can have a large dependence on the chemistry of the lipid in modulating membrane protein structure and function, including allosterically modulating protein-protein interactions.

\section{Methods}

Purification of AmtB-GInK complex. AmtB wild-type and Strep-tag II-tagged $\mathrm{GlnK}(\operatorname{stGlnK})$ was expressed and purified as previously described ${ }^{14}$, 31 . In brief, stGlnK was expressed from pET28b (Novagen) in Escherichia coli ArcticExpress (DE3) RIL (Agilent Technologies), and protein expression was induced with 0.1 $\mathrm{mM}$ 1-thio- $\beta$-D-galactopyranoside (IPTG) and grown overnight at $20^{\circ} \mathrm{C}$. Cell pellets of GlnK were resuspended in NHA buffer $(50 \mathrm{mM}$ Tris $\mathrm{pH} 7.4$ at room temperature, $300 \mathrm{mM}$ sodium chloride, $20 \mathrm{mM}$ imidazole, and $10 \%$ glycerol). The cells were lysed with $4-5$ passes through a Microfluidics M-110P microfluidizer at $20,000 \mathrm{psi}$ and then clarified by centrifugation $\left(30 \mathrm{~min}\right.$ at $30,000 \times g$ at $\left.4^{\circ} \mathrm{C}\right)$. The filtered supernatant containing recombinant Strep-tag II-tagged GlnK was then applied onto a StrepTrap HP $5 \mathrm{~mL}$ column (GE Healthcare) and eluted with the same buffer containing $2.5 \mathrm{mM}$ D-desthiobiotin. The peak fractions containing Strep-tag II-tagged GlnK were pooled, concentrated, and loaded onto a HiLoad 16/ 600 Superdex $75 \mathrm{pg}$ column (GE Healthcare) equilibrated in GF buffer $(20 \mathrm{mM}$ Tris $\mathrm{pH} 7.4$ at room temperature, $100 \mathrm{mM}$ sodium chloride, and $10 \%$ glycerol). AmtB was expressed as TEV protease cleavable $\mathrm{N}$-terminal fusion to maltose-binding protein (MBP) preceded by a secretion signal peptide (pelB) and 10x His-tag from pET15b (Novagen) in Escherichia coli C41(DE3) (Lucigen). Expression of AmtB was induced with $0.5 \mathrm{mM}$ IPTG and cells were grown overnight at $20^{\circ} \mathrm{C}$. Cell pellets of AmtB were resuspended and lyszed as described for stGlnK. Membranes were pelleted by centrifugation $\left(2 \mathrm{~h}\right.$ at $100,000 \times g$ at $\left.4{ }^{\circ} \mathrm{C}\right)$ and $\mathrm{AmtB}$ was extracted overnight with $5 \%$ octyl glucoside. The supernatant was filtered before loading onto a $5 \mathrm{~mL}$ HisTrap-HP column (GE Healthcare) equilibrated in buffer NHA-DDM (200 mM sodium chloride, $10 \%$ glycerol, $20 \mathrm{mM}$ imidazole, $0.025 \% \mathrm{DDM}$ and 50 $\mathrm{mM}$ Tris $\mathrm{pH} 7.4$ at room temperature). After the clarified supernatant was loaded, the column was initially washed with $40-50 \mathrm{~mL}$ of NHA-DDM supplemented with $1 \%$ OG followed by several column volumes of NHA-DDM until a steady baseline was reached. AmtB was eluted with a linear gradient to $100 \%$ in two column volumes of NHB-DDM ( $100 \mathrm{mM}$ sodium chloride, $10 \%$ glycerol, $500 \mathrm{mM}$ imidazole, $0.025 \%$ DDM and $50 \mathrm{mM}$ Tris $\mathrm{pH} 7.4$ at room temperature). Peak fractions were pooled and injected onto a HiPrep 26/10 desalting column (GE Healthcare) equilibrated in NHA-DDM. Peak fractions were pooled and supplemented with 5 $\mathrm{mM} 2$-mercaptoethanol (BME) and His-tagged TEV and incubated overnight at $4^{\circ}$ C. After overnight incubation, the sample was filtered and passed back over a $5 \mathrm{~mL}$ HisTrap HP column equilibrated in NHA-DDM. Flow-through containing the untagged AmtB was collected and concentrated using a $100 \mathrm{kDa}$ MWCO concentrator. The Y51F point mutation was introduced into $\mathrm{GlnK}$ as this residue can be modified with uridine by uridylyl transferase $\mathrm{Gln} D$ and prevent the interaction with $A m B^{8,12,48}$. Unless otherwise stated, all purification steps were carried out at $4{ }^{\circ} \mathrm{C}$. To generate the $\mathrm{AmtB}-\mathrm{G} \operatorname{lnK}$ complex, purified tagless $\mathrm{AmtB}$ and stGlnK were mixed at a molar ratio of 1:3 in GF buffer $(50 \mathrm{mM}$ Tris $\mathrm{pH} 7.4$ at room temperature, $100 \mathrm{mM}$ sodium chloride, $10 \%$ glycerol, $0.025 \%$ DDM, and $1 \mathrm{mM}$ adenosine diphosphate (ADP)) and incubated overnight. The mixture of AmtB and stGlnK was loaded onto a StrepTrap HP $5 \mathrm{~mL}$ column (GE Healthcare) to capture both $\mathrm{AmtB}$ in complex with stGlnK and stGlnK, and flow through contained nonfunctional AmtB. Bound protein was eluted with the same buffer containing 2.5 mM D-desthiobiotin, concentrated, and loaded onto a Superdex 200 Increase 10/ $300 \mathrm{GL}$ column (GE Healthcare) equilibrated in GF buffer supplemented with $0.5 \%$ $\mathrm{C}_{8} \mathrm{E}_{4}$ instead of DDM. Peak fractions containing the AmtB-stGlnK were pooled, concentrated, flash frozen in liquid nitrogen, and stored at $-80^{\circ} \mathrm{C}$.

Protein quantification. Soluble and membrane protein concentration in the buffer not containing ADP was determined with the DC Protein Assay kit (Bio-Rad) using bovine serum albumin as the standard. For quantification of samples containing ADP, which interferes with protein assays, aliquots of the purified AmtB-GlnK complex were thawed at room temperature, immediately mixed with 0.25 volumes of $5 \times$ Laemmli sample buffer ${ }^{49}$, and incubated at room temperature for $30 \mathrm{~min}$. Heating was avoided as it promotes aggregation of $\mathrm{AmtB}$. The prepared samples were loaded on a precast $12 \%$ acrylamide gel (TGX, 10 well, BioRad). Included on each gel was one well with pre-stained size markers (BioRad), and at least two wells loaded with a standard mixture of purified AmtB and stGlnK at a molar ratio of 1:1. Gels were run at $60 \mathrm{~V}$ during stacking and at $100 \mathrm{~V}$ once sample was in the resolving gel at room temperature. After separation, each gel was rinsed in water for $15 \mathrm{~min}$, stained with $0.025 \%$ Coomassie brilliant blue R-250 (Sigma B0149 ) in $10 \%$ acetic acid using a rapid, hot-staining protocol ${ }^{50}$ followed destaining overnight in a $100 \mathrm{~mL}$ portion of $10 \%$ acetic acid. General procedures for quantitative densitometry followed the recommendations of Gassmann and colleagues $^{51}$. The gel was placed on a Ricoh 4504 scanner, imaged in transmittance mode at a resolution of $400 \mathrm{dpi}$, and stored as a 16-bit greyscale TIFF image. For densitometry analysis, each file was opened in Image $\mathrm{J}^{52}$ and the original greyscale values were converted to optical density $(\mathrm{OD})$ values. Individual full-width lanes were then defined, their OD profiles calculated, and the integrated stain density summed for the $A m t B$ and GlnK bands. The amount of $A m t B$ or $G \operatorname{lnK}$ protein in each lane was calculated from the stain density in the lane, with reference to the average stain density of the AmtB or GlnK band in lanes loaded with the known standard mix of AmtB and GlnK. The protein concentration for AmtB and GlnK was the average of the values determined from at least two separately Coomassiestained gels.

Surface plasmon resonance. SPR experiments were performed using a Biacore 3000 optical biosensor (GE Healthcare/Biacore AB) with CM3 sensor chip. Immobilization of $\mathrm{GlnK}$ was conducted at $25^{\circ} \mathrm{C}$ using amine coupling kit (GE Healthcare) at a flow rate of $10 \mu \mathrm{L} \mathrm{min}^{-1}$ in PBS $\left(8.06 \mathrm{mM} \mathrm{Na}_{2} \mathrm{HPO}_{4}\right.$ and $1.94 \mathrm{mM}$ $\mathrm{KH}_{2} \mathrm{PO}_{4}, 2.7 \mathrm{mM} \mathrm{KCl}, 137 \mathrm{mM} \mathrm{NaCl}, \mathrm{pH}$ 7.4). In order to generate low-density GlnK sensor, the chip surface was pretreated by sequential injection of $40 \mu \mathrm{L}$ of activation solution (1:1 mixture of $0.4 \mathrm{M} \mathrm{EDC}$ and $0.1 \mathrm{M} \mathrm{NHS}$ ) and $40 \mu \mathrm{L}$ of deactivation solution ( $1.0 \mathrm{M}$ ethanolamine, $\mathrm{pH}$ 8.5). The pretreated surface was activated again for $2 \mathrm{~min}$ followed by $1 \mathrm{~min}$ injection of stGlnK in buffer $(2.5-5 \mu \mathrm{g}$ $\mathrm{ml}^{-1}$ in $10 \mathrm{mM}$ sodium acetate, $\mathrm{pH} 5.5$ ), and then washed with $200 \mathrm{mM}$ ammonium acetate $(\mathrm{pH}$ 7.4) until no further decline in signal response. Using this procedure, GlnK surface with different densities ranging from 150 to $400 \mathrm{RU}$ was produced. A flow cell with the same treatment but without coupled protein was used as a reference surface. The sensor chip was further stabilized with 10 injections of binding buffer $(200 \mathrm{mM}$ ammonium acetate, $\mathrm{pH} 7.4,50 \mu \mathrm{M}$ ADP, $0.5 \%$ $\mathrm{C}_{8} \mathrm{E}_{4}$ ) followed by $1 \mathrm{~h}$ of continuous flow at $30 \mu \mathrm{L} \mathrm{min}^{-1}$. Binding experiments were performed at a flow rate of $30 \mu \mathrm{L} \mathrm{min}^{-1}$ at different temperatures $(13,17,21,25$, and $29^{\circ} \mathrm{C}$ ) using freshly buffer-exchanged $\mathrm{AmtB}$ in binding buffer at a concentration range of $0.05-1.4 \mu \mathrm{M}$. Reference and buffer corrected SPR responses were collected, and the data were analyzed using Biacore evaluation software. The kinetic parameters and $K_{\mathrm{D}}$ value were determined for each temperature by fitting the sensorgram series to a Langmuir 1:1 binding model.

Preparation and titration of ADP or phospholipids. A stock of ADP was prepared by dissolving the ammonium salt form of ADP in water followed by adjusting the $\mathrm{pH}$ with ammonium hydroxide to 7.0. Serial dilutions into MS buffer (200 $\mathrm{mM}$ ammonium acetate, $0.5 \% \mathrm{C} 8 \mathrm{E} 4$, and $\mathrm{pH}$ adjusted to 7.4 with ammonium hydroxide) supplemented with $5 \mathrm{mM} \mathrm{BME}$ was performed to prepare ADP at certain concentrations. Phospholipids were prepared as previously described ${ }^{31}$. In brief, stock solutions of each phospholipid were resuspended in chloroform, aliqouted, and chloroform was removed by a steady stream on nitrogen gas. Lipid films were placed under vacuum overnight to prepare to resuspend in MS buffer supplemented with $5 \mathrm{mM} 2$-mercaptoethanol. Phospholipid concentration was determined by phosphorus analysis.

Preparation of AmtB-GInK complex for native MS. A frozen aliquot of purified AmtB-GlnK complex was thawed on ice prior to buffer exchange into MS buffer supplemented with a desired concentration of ADP following established methods $^{53}$

Native MS. Native MS was performed on a Synapt G1 HDMS instrument (Waters corporation) with a $32 \mathrm{k}$ RF generator. AmtB-GlnK samples were at a final concentration of $2 \mu \mathrm{M}$. Instrument parameters were tuned to maximize ion intensity and simultaneously preserve the native-like state of AmtB. The instrument was set to a capillary voltage of $1.7 \mathrm{kV}$, sampling cone voltage of $100 \mathrm{~V}$, extractor cone voltage of $10 \mathrm{~V}$, and argon flow rate at $7 \mathrm{~mL} \mathrm{~min}^{-1}\left(5.2 \times 10^{-2} \mathrm{mbar}\right)$. The T-wave settings for trap $\left(300 \mathrm{~ms}^{-1} / 2.0 \mathrm{~V}\right)$, IMS $\left(300 \mathrm{~ms}^{-1} / 20 \mathrm{~V}\right)$, and transfer $\left(100 \mathrm{~ms}^{-1} / 10\right.$ $\mathrm{V})$, source temperature $\left(110^{\circ} \mathrm{C}\right)$ trap collision voltage $(20 \mathrm{~V})$ and transfer collision voltage $(160 \mathrm{~V})$ and trap bias $(35 \mathrm{~V})$ were also optimized.

Native MS data analysis. Native MS data were processed using the software program Pulsar ${ }^{54}$ and deconvoluted using Unidec ${ }^{55}$ software with the following settings: no smoothing, $m / z$ range $6000-12,000$, charge range 10-30, mass sampling of $5 \mathrm{Da}$, and peak FWHM of 1 . The intensities of apo AmtB $(A)$ and $\mathrm{AmtB}-\mathrm{G} \operatorname{lnK}(\mathrm{AG})$ and $\mathrm{AmtB}\left(\mathrm{AL}_{n}\right)$ and $\mathrm{AmtB}-\mathrm{GlnK}\left(\mathrm{AGL}_{n}\right)$ bound to $\mathrm{L}_{n} \operatorname{lipid}(\mathrm{s})$ were converted to mole fraction for a given lipid titration. Notably, the non-specific ADP adducted peaks of AmtB and AmtB-GlnK and those bound to lipid(s) were taken into account when converting to mole fraction. The interaction between $\mathrm{AL}_{n}$ and $\operatorname{GlnK}(G)$ is dependent on the apparent equilibrium association constant $\left(K_{\mathrm{A}}\right.$ $\mathrm{AL} n \mathrm{G})$ :

$$
K_{\mathrm{A}, \mathrm{AL} n \mathrm{G}}=\frac{\left[\mathrm{AL}_{n} G\right]}{\left[\mathrm{AL}_{n}\right][G]}
$$


Where $n$ is an integer starting at 0 in the case of apo. And for a lipid binding to A:

$$
K_{\mathrm{A}, \mathrm{AL} n}=\frac{\left[\mathrm{AL}_{n}\right]}{\left[\mathrm{AL}_{n-1}\right][L]}
$$

And binding event to AG:

$$
K_{\mathrm{A}, \mathrm{AGL} n}=\frac{\left[\mathrm{AGL}_{\mathrm{n}}\right]}{\left[\mathrm{AGL}_{\mathrm{n}-1}\right][L]}
$$

The total AmtB $\left(A_{\text {total }}\right)$ in the system can be represented as:

$$
\left[A_{\text {total }}\right]=[A]+\sum_{i=1}^{n}\left[\mathrm{AL}_{i}\right]+[\mathrm{AG}]+\sum_{i=1}^{n}\left[\mathrm{AGL}_{i}\right]
$$

substituting Eqs. (1-3) into (4):

$$
\begin{array}{r}
{\left[A_{\text {total }}\right]=[A]+\sum_{i=1}^{n}[A][L]^{i} \prod_{j=1}^{i} K_{\mathrm{A}, \mathrm{AL} j}+K_{\mathrm{A}, \mathrm{AL} \mathrm{L}_{0} \mathrm{G}}[A][G]} \\
+\sum_{i=1}^{n} K_{\mathrm{A}, \mathrm{AL} i \mathrm{G}}[A][\mathrm{G}][L]^{i} \prod_{j=1}^{i} K_{\mathrm{A}, \mathrm{AL} j}
\end{array}
$$

Equation (5) can be rearranged to calculate the mole fraction of $\operatorname{AL} n\left(F_{\mathrm{AL} n}\right)$ :

$$
F_{\mathrm{AL} n}=\frac{[L]^{n} \prod_{j=1}^{n} K_{\mathrm{A}, \mathrm{AL} j}}{\left[A_{\text {total }}\right]}
$$

And for AGLn $\left(\mathrm{F}_{\mathrm{AGL} n}\right)$ :

$$
F_{\mathrm{AGL} n}=\frac{K_{\mathrm{A}, \mathrm{AL} n \mathrm{G}}[G][L]^{n} \prod_{j=1}^{n} K_{\mathrm{A}, \mathrm{AL} j}}{\left[A_{\mathrm{total}}\right]}
$$

The free concentration of $G$ was calculated as follows:

$$
[G]=\left[G_{\text {total }}\right]-\left\{\left[A_{\text {total }}\right] \cdot\left(F_{\mathrm{AG}}+\sum_{i=1}^{n} F_{\mathrm{AGL} i}\right)\right\}
$$

And for the free concentration of $L$ :

$$
[L]=\left[L_{\text {total }}\right]-\left\{\left[A_{\text {total }}\right] \cdot\left(\sum_{i=1}^{n} i F_{\mathrm{AL} i}+\sum_{i=1}^{n} i F_{\mathrm{AGL} i}\right)\right\}
$$

The above binding model (Fig. 3) was coded into Python (http://www.python.org) and made use of libraries scipy ${ }^{56}$, numpy ${ }^{57}$, and matplotlib ${ }^{58}$. The binding model was globally fit to the mole fraction data collected at a given temperature through minimization of the pseudo- $\chi^{2}$ function:

$$
\chi^{2}=\sum_{i=0}^{n} \sum_{j=0}^{d}\left(F_{\mathrm{AL} i_{j}}^{\exp }-F_{\mathrm{AL}_{i_{j}}}^{\text {calc }}\right)^{2}+\left(F_{\mathrm{AGL} i_{j}}^{\exp }-F_{\mathrm{AGL}_{i_{j}}}^{\mathrm{calc}}\right)^{2}
$$

where $n$ is the total number of lipids bound and $d$ is the total number of data points.

In the above binding model, all equilibrium association constants are treated as a separate variable in the fitting routine. The coupling factor $\left(\alpha_{i}\right)$ for a given number of lipids bound (i) was calculated as follows:

$$
\propto_{i}=\frac{K_{\mathrm{A}, \mathrm{AGL} i}}{K_{\mathrm{A}, \mathrm{AGL}(i-1)}}
$$

Thermodynamic parameters for the AmtB-GlnK complex in the absence of lipid were determined using the nonlinear form of the "van't" Hoff equation: ${ }^{59}$

$$
\ln \left(K_{\mathrm{A}}\right)=\frac{\Delta H_{T_{\mathrm{o}}}-T_{\mathrm{o}} \Delta C_{\mathrm{p}}}{R}\left(\frac{1}{T_{\mathrm{o}}}-\frac{1}{\mathrm{~T}}\right)+\frac{\Delta C_{\mathrm{p}}}{R} \ln \left(\frac{T}{T_{\mathrm{o}}}\right)+\ln \left(K_{\mathrm{o}}\right)
$$

where $K_{\mathrm{A}}$ is the equilibrium association constant, $K_{\mathrm{o}}$ is the equilibrium association constant at the reference temperature $\left(T_{\mathrm{o}}\right), \Delta H$ is the standard enthalpy at $T_{\mathrm{o}}, \Delta C_{\mathrm{p}}$ is the change in heat capacity at constant pressure, and $R$ is the universal gas constant. The average and standard error of the mean was determined by fitting the above equation coded into Python to experimental data for each replicate with $T_{\mathrm{o}}$ equal to $298 \mathrm{~K}$

Data availability. The data that support the findings of this study are available within the article and Supplementary Information. Other data and binding model Python code are available from the corresponding author on reasonable request.
Received: 23 August 2017 Accepted: 22 November 2017

Published online: 19 December 2017

\section{References}

1. Davis, M. J. et al. Regulation of ion channels by protein tyrosine phosphorylation. Am. J. Physiol. Heart Circ. Physiol. 281, H1835-H1862 (2001).

2. Tillman, T. S. \& Cascio, M. Effects of membrane lipids on ion channel structure and function. Cell. Biochem. Biophys. 38, 161-190 (2003).

3. Gamper, N. \& Shapiro, M. S. Regulation of ion transport proteins by membrane phosphoinositides. Nat. Rev. Neurosci. 8, 921-934 (2007).

4. Rosenhouse-Dantsker, A., Mehta, D. \& Levitan, I. Regulation of ion channels by membrane lipids. Compr. Physiol. 2, 31-68 (2012).

5. Hansen, S. B. Lipid agonism: the PIP2 paradigm of ligand-gated ion channels Biochim. Biophys. Acta 1851, 620-628 (2015).

6. Thomas, G. H., Mullins, J. G. \& Merrick, M. Membrane topology of the Mep/ Amt family of ammonium transporters. Mol. Microbiol. 37, 331-344 (2000).

7. Arcondeguy, T., Jack, R. \& Merrick, M. P(II) signal transduction proteins, pivotal players in microbial nitrogen control. Microbiol. Mol. Biol. Rev. 65, 80-105 (2001).

8. Durand, A. \& Merrick, M. In vitro analysis of the Escherichia coli AmtB-GlnK complex reveals a stoichiometric interaction and sensitivity to ATP and 2oxoglutarate. J. Biol. Chem. 281, 29558-29567 (2006).

9. Radchenko, M. V., Thornton, J. \& Merrick, M. Control of AmtB-GlnK complex formation by intracellular levels of ATP, ADP, and 2-oxoglutarate. J. Biol. Chem. 285, 31037-31045 (2010).

10. Radchenko, M. V., Thornton, J. \& Merrick, M. Association and dissociation of the GlnK-AmtB complex in response to cellular nitrogen status can occur in the absence of GlnK post-translational modification. Front. Microbiol. 5, 731 (2014).

11. Conroy, M. J. et al. The crystal structure of the Escherichia coli AmtB-GlnK complex reveals how GlnK regulates the ammonia channel. Proc. Natl Acad. Sci. USA 104, 1213-1218 (2007).

12. Gruswitz, F., O'Connell, J. 3rd \& Stroud, R. M. Inhibitory complex of the transmembrane ammonia channel, AmtB, and the cytosolic regulatory protein, GlnK, at 1.96 A. Proc. Natl Acad. Sci. USA 104, 42-47 (2007).

13. Berman, H. M. et al. The protein data bank. Nucleic Acids Res. 28, 235-242 (2000).

14. Laganowsky, A. et al. Membrane proteins bind lipids selectively to modulate their structure and function. Nature 510, 172-175 (2014).

15. Gulbakan, B., Barylyuk, K. \& Zenobi, R. Determination of thermodynamic and kinetic properties of biomolecules by mass spectrometry. Curr. Opin. Biotechnol. 31, 65-72 (2015).

16. Loo, J. A. Studying noncovalent protein complexes by electrospray ionization mass spectrometry. Mass. Spectrom. Rev. 16, 1-23 (1997).

17. Marcoux, J. \& Robinson, C. V. Twenty years of gas phase structural biology. Structure 21, 1541-1550 (2013).

18. Pacholarz, K. J., Garlish, R. A., Taylor, R. J. \& Barran, P. E. Mass spectrometry based tools to investigate protein-ligand interactions for drug discovery. Chem Soc. Rev. 41, 4335-4355 (2012).

19. Dyachenko, A., Gruber, R., Shimon, L., Horovitz, A. \& Sharon, M. Allosteric mechanisms can be distinguished using structural mass spectrometry. Proc. Natl Acad. Sci. USA 110, 7235-7239 (2013).

20. Cubrilovic, D. et al. Determination of protein-ligand binding constants of a cooperatively regulated tetrameric enzyme using electrospray mass spectrometry. ACS Chem. Biol. 9, 218-226 (2014).

21. Hilton, G. R. \& Benesch, J. L. Two decades of studying non-covalent biomolecular assemblies by means of electrospray ionization mass spectrometry. J. R. Soc. Interface 9, 801-816 (2012).

22. Deng, L., Kitova, E. N. \& Klassen, J. S. Dissociation kinetics of the streptavidinbiotin interaction measured using direct electrospray ionization mass spectrometry analysis. J. Am. Soc. Mass. Spectrom. 24, 49-56 (2013).

23. Robinson, C. V. From molecular chaperones to membrane motors: through the lens of a mass spectrometrist. Biochem. Soc. Trans. 45, 251-260 (2017).

24. Barrera, N. P. et al. Mass spectrometry of membrane transporters reveals subunit stoichiometry and interactions. Nat. Methods 6, 585-587 (2009).

25. Zhou, M. et al. Mass spectrometry of intact V-type ATPases reveals bound lipids and the effects of nucleotide binding. Science 334, 380-385 (2011).

26. Marcoux, J. et al. Mass spectrometry reveals synergistic effects of nucleotides, lipids, and drugs binding to a multidrug resistance efflux pump. Proc. Natl Acad. Sci. USA 110, 9704-9709 (2013).

27. Housden, N. G. et al. Intrinsically disordered protein threads through the bacterial outer-membrane porin OmpF. Science 340, 1570-1574 (2013).

28. Gault, J. et al. High-resolution mass spectrometry of small molecules bound to membrane proteins. Nat. Methods 13, 333-336 (2016).

29. Gupta, K. et al. The role of interfacial lipids in stabilizing membrane protein oligomers. Nature 541, 421-424 (2017). 
30. El-Baba, T. J. et al. Melting proteins: evidence for multiple stable structures upon thermal denaturation of native ubiquitin from ion mobility spectrometrymass spectrometry measurements. J. Am. Chem. Soc. 139, 6306-6309 (2017).

31. Cong, X. et al. Determining membrane protein-lipid binding thermodynamics using native mass spectrometry. J. Am. Chem. Soc. 138, 4346-4349 (2016).

32. Daneshfar, R., Kitova, E. N. \& Klassen, J. S. Determination of protein-ligand association thermochemistry using variable-temperature nanoelectrospray mass spectrometry. J. Am. Chem. Soc. 126, 4786-4787 (2004).

33. Reading, E. et al. The role of the detergent micelle in preserving the structure of membrane proteins in the gas phase. Angew. Chem. Int. Ed. Engl. 54, 4577-4581 (2015)

34. van 't Hoff, M. J. H. Etudes de dynamique chimique. Recl. Des. Trav. Chim. Des. Pays-Bas 3, 333-336 (1884).

35. Naghibi, H., Tamura, A. \& Sturtevant, J. M. Significant discrepancies between van 't Hoff and calorimetric enthalpies. Proc. Natl Acad. Sci. USA 92, 5597-5599 (1995).

36. Prabhu, N. V. \& Sharp, K. A. Heat capacity in proteins. Annu. Rev. Phys. Chem. 56, 521-548 (2005).

37. Krissinel, E. \& Henrick, K. Inference of macromolecular assemblies from crystalline state. J. Mol. Biol. 372, 774-797 (2007).

38. Eftink, M. R., Anusiem, A. C. \& Biltonen, R. L. Enthalpy-entropy compensation and heat capacity changes for protein-ligand interactions: general thermodynamic models and data for the binding of nucleotides to ribonuclease A. Biochemistry 22, 3884-3896 (1983).

39. Thompson, M. \& Woodbury, N. W. Thermodynamics of specific and nonspecific DNA binding by two DNA-binding domains conjugated to fluorescent probes. Biophys. J. 81, 1793-1804 (2001).

40. Makhatadze, G. I. \& Privalov, P. L. Heat capacity of proteins. I. Partial molar heat capacity of individual amino acid residues in aqueous solution: hydration effect. J. Mol. Biol. 213, 375-384 (1990).

41. Marty, M. T. et al. Bayesian deconvolution of mass and ion mobility spectra: from binary interactions to polydisperse ensembles. Anal. Chem. 87, 4370-4376 (2015).

42. De Siervo, A. J. Alterations in the phospholipid composition of Escherichia coli B during growth at different temperatures. J. Bacteriol. 100, 1342-1349 (1969).

43. Zhang, Y. M. \& Rock, C. O. Membrane lipid homeostasis in bacteria. Nat. Rev. Microbiol. 6, 222-233 (2008).

44. Cronan, J. E. Jr. \& Gelmann, E. P. Physical properties of membrane lipids: biological relevance and regulation. Bacteriol. Rev. 39, 232-256 (1975).

45. Monod, J., Wyman, J. \& Changeux, J. P. On the nature of allosteric transitions: a plausible model. J. Mol. Biol. 12, 88-118 (1965).

46. Motlagh, H. N., Wrabl, J. O., Li, J. \& Hilser, V. J. The ensemble nature of allostery. Nature 508, 331-339 (2014).

47. Liu, J. \& Nussinov, R. Allostery: an overview of its history, concepts, methods, and applications. PLoS Comput. Biol. 12, e1004966 (2016).

48. Javelle, A., Severi, E., Thornton, J. \& Merrick, M. Ammonium sensing in Escherichia coli. Role of the ammonium transporter AmtB and AmtB-GlnK complex formation. J. Biol. Chem. 279, 8530-8538 (2004).

49. Laemmli, U. K. Cleavage of structural proteins during the assembly of the head of bacteriophage T4. Nature 227, 680-685 (1970).

50. Westermeier, R. Sensitive, quantitative, and fast modifications for Coomassie Blue staining of polyacrylamide gels. Proteomics 6, 61-64 (2006).

51. Gassmann, M., Grenacher, B., Rohde, B. \& Vogel, J. Quantifying western blots: pitfalls of densitometry. Electrophoresis 30, 1845-1855 (2009).

52. Schneider, C. A., Rasband, W. S. \& Eliceiri, K. W. NIH Image to ImageJ: 25 years of image analysis. Nat. Methods 9, 671-675 (2012).

53. Laganowsky, A., Reading, E., Hopper, J. T. \& Robinson, C. V. Mass spectrometry of intact membrane protein complexes. Nat. Protoc. 8, 639-651 (2013).
54. Allison, T. M. et al. Quantifying the stabilizing effects of protein-ligand interactions in the gas phase. Nat. Commun. 6, 8551 (2015).

55. Reading, E. et al. The effect of detergent, temperature, and lipid on the oligomeric state of mscl constructs: insights from mass spectrometry. Chem. Biol. 22, 593-603 (2015).

56. Millman, K. J. \& Aivazis, M. Python for scientists and engineers. Comput. Sci. Eng. 13, 9-12 (2011).

57. van der Walt, S., Colbert, S. C. \& Varoquaux, G. The NumPy array: a structure for efficient numerical computation. Comput. Sci. Eng. 13, 22-30 (2011).

58. Hunter, J. D. Matplotlib: A 2D graphics environment. Comput. Sci. Eng. 9, 90-95 (2007).

59. Liu, Y. \& Sturtevant, J. M. The observed change in heat capacity accompanying the thermal unfolding of proteins depends on the composition of the solution and on the method employed to change the temperature of unfolding. Biochemistry 35, 3059-3062 (1996).

\section{Acknowledgements}

We thank Dr Magnus Höök (Texas A\&M Health Science Center) and Dr David Russell (Texas A\&M University) for useful discussion. This work was supported by new faculty startup funds from the Institute of Biosciences and Technology, Texas A\&M Health Science Center, and the Department of Chemistry, Texas A\&M University, and partial support from National Institute of General Medical Sciences of the National Institutes of Health under Award Number DP2GM123486.

\section{Author contributions}

X.C. and A.L. designed the research. All authors performed the experiments. X.C., X.L., and A.L. analyzed the data. X.C and A.L. wrote the paper with input from the other authors.

\section{Additional information}

Supplementary Information accompanies this paper at https://doi.org/10.1038/s41467017-02397-0.

Competing interests: The authors declare no competing financial interests.

Reprints and permission information is available online at http://npg.nature.com/ reprintsandpermissions/

Publisher's note: Springer Nature remains neutral with regard to jurisdictional claims in published maps and institutional affiliations.

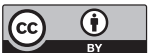

Open Access This article is licensed under a Creative Commons Attribution 4.0 International License, which permits use, sharing, adaptation, distribution and reproduction in any medium or format, as long as you give appropriate credit to the original author(s) and the source, provide a link to the Creative Commons license, and indicate if changes were made. The images or other third party material in this article are included in the article's Creative Commons license, unless indicated otherwise in a credit line to the material. If material is not included in the article's Creative Commons license and your intended use is not permitted by statutory regulation or exceeds the permitted use, you will need to obtain permission directly from the copyright holder. To view a copy of this license, visit http://creativecommons.org/ licenses/by/4.0/.

(C) The Author(s) 2017 